\title{
Regard sur les lazarets en terre canadienne
}

\author{
Jean Milot MD FRCSC
}

\section{J Milot. Regard sur les lazarets en terre canadienne. Can J Infect Dis Med Microbiol 2008;19(2):193-196.}

Puisant dans les nombreuses références qu'offrent les publications médicales canadiennes du milieu du XIX ${ }^{\mathrm{e}}$ siècle à nos jours, l'auteur fait découvrir l'existence de lazarets en terre canadienne, décrit l'impact de la maladie sur les conditions de vie des lépreux qui y étaient confinés et en souligne les contrecoups tant sur le plan physique et psychologique que social. Il présente un bref aperçu de la maladie, ses symptômes, ses signes ainsi que ses complications oculaires et rappelle les premiers moyens thérapeutiques à base d'huile de chaulmoogra introduits dans la colonie de Tracadie vers 1901. Il illustre son propos en évoquant la vie dans les lazarets de l'île de Sheldrake (1844-1848) et de Tracadie (1848-1965) au Nouveau-Brunswick, puis dans ceux des îles D'Arcy (1891-1924) et de Bentinck (1924-1957) en Colombie-Britannique.

\section{A look at leprosaria on Canadian territory}

Canadian medical journals from the mid-19th century to the present day are used to illustrate the history and evolution of lazarettos in Canada. The living conditions in these 'leper colonies' and their psychological, social and physical impacts are depicted. The present article reviews the cause, signs and symptoms of leprosy, and highlights the frequent ocular complications, as well as the use of chaulmoogra oil in Tracadie, New Brunswick, around 1901. The history of the four lazarettos in Canada Sheldrake Island (1844 to 1848) and Tracadie (1848 to 1965) in New Brunswick, and D'Arcy Island (1891 to 1924) and Bentinck Island (1924 to 1957) in British Columbia - is described.

Mots-clef: Lazaret; Lèpre; Léproserie

a lèpre, ou maladie de Hansen, fait sur Terre des ravages qui Lremontent à la nuit des temps. La Bible, plus précisément le Livre des Rois dans l'Ancien Testament, nous apprend que la lèpre existait déjà sur les rives de la Méditerranée. Naaman le Syrien, chef de l'armée du roi, en était affligé. Sous la même rubrique, le papyrus d'Ebers, un des plus anciens traités médicaux qui nous soient parvenus et qui a été rédigé entre l'an 1600 et 1500 avant notre ère, mentionne la lèpre parmi les nombreuses maladies qui sévissaient en Égypte. Au même titre, dans l'Évangile selon saint Luc $(5,14)$ : « un lépreux vint trouver Jésus... Pris de pitié devant cet homme, Jésus étendit la main, le toucha. À l'instant même, sa lèpre le quitta ». Dans le même Évangile (Luc, 16, 20) : « un pauvre, nommé Lazare, ami de Jésus, était couché à sa porte, couvert d'ulcères, et même les chiens venaient encore lécher ses ulcères ». Voilà l'origine du terme « lazaret » pour désigner les léproseries. Puis, que dire des croisés qui favorisèrent à leur insu la propagation de la lèpre en l'Europe et au Moyen-Orient, à l'aller et au retour (1) ? Enfin, au Moyen Âge, les misérables lépreux, inspirant horreur et terreur, devaient agiter une crécelle pour annoncer leur approche et écarter les gens sur leur passage.

On croit que la lèpre n'aurait fait son apparition dans le Nouveau Monde que peu de temps après l'arrivée de Christophe Colomb, en 1492. Ce fut une affreuse malédiction apportée par les colonisateurs venus du Portugal et d'Espagne. où sévissait déjà la maladie. Celle-ci s'abattit sur la population amérindienne de l'Amérique du Sud et des Antilles, pour prendre des proportions endémiques.

\section{MANIFESTATIONS CLINIQUES ET TRAITEMENTS DE LA LÈPRE}

Quel aspect cette maladie infectieuse et contagieuse peut-elle prendre, même de nos jours ? Cette maladie transmissible demeure encore aujourd'hui endémique en Asie, en Afrique, mais aussi en Amérique du Sud et dans les Antilles. L'agent étiologique est le Mycobacterium leprae, bacille qui fut découvert et isolé en 1869 par un médecin et biologiste norvégien, Henrik Armauer Hansen (1841-1912). Cet agent pathogène a une affinité particulière pour la peau, les os et les nerfs périphériques. L'homme demeure vraisemblablement la source d'infection, et le mode de contagion semble être le contact prolongé et étroit avec les lépromateux. Cependant, la majorité des personnes possèdent une résistance naturelle visà-vis du bacille de Hensen. Le début de la manifestation clinique est insidieux, avec une incubation de quelques semaines à plusieurs années avant l'apparition des premiers signes et symptômes. Au début, le patient ressent une sensation de démangeaison et de fourmillement de la peau. Malgré la confusion considérable des publications médicales au sujet de la classification des divers types de lèpres, on reconnaît qu'il en existerait deux formes : la forme lépromateuse ou cutanée, et la forme tuberculoïde ou nerveuse. La première se manifeste par des nodules anesthésiques et des lépromes infiltrant la peau et les muqueuses, accompagnés de douleurs périphériques. Dans la seconde, les nerfs sont distendus par des nodules accompagnés d'algies ou de zones d'anesthésie et d'atrophie fréquentes des mains.

De toutes les maladies systémiques, la lèpre a un taux de localisation oculaire secondaire des plus élevés, pouvant atteindre $91 \%$ (2). Ainsi, les complications oculaires de la maladie de Hansen sont extrêmement fréquentes, et la cornée semble la plus vulnérable, apparaissant saupoudrée d'une poussière de craie ou de farine. Les autres complications oculaires observées sont l'anesthésie cornéenne, l'iritis, la présence de lépromes extrêmement fins de la dimension d'une tête d'épingle sur l'iris, la perte des cils et des sourcils. Le

L'Université de Montréal, Montréal, Québec

Correspondence: Dr Jean Milot, Hôpital Sainte-Justine, 3175, ch. de la Côte-Sainte-Catherine, Montréal, Québec, H3T 1 C5.

Telephone 514-345 4715, fax 514-345-7706, e-mail jeanmilot@videotron.ca

Received for publication July 27, 2007. Accepted November 2, 2007 


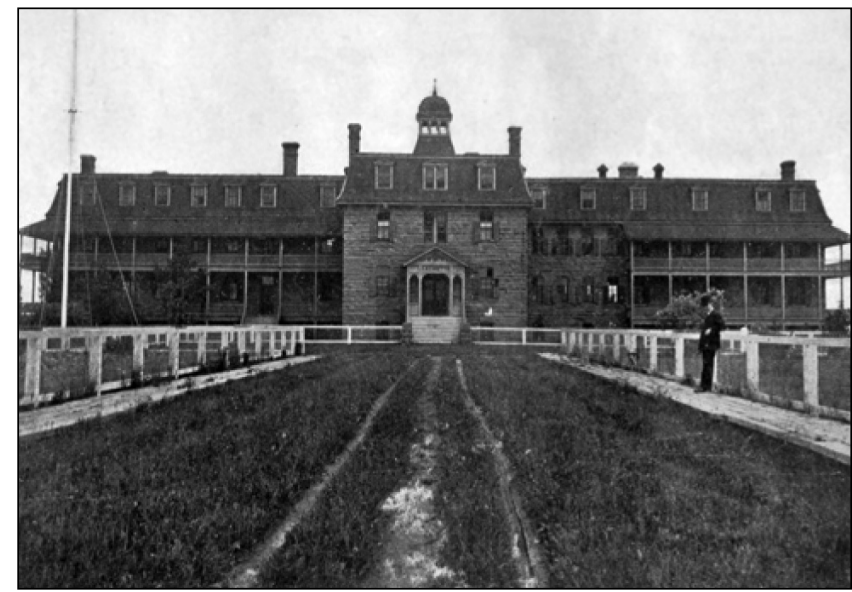

Figure 1) Lazaret à Tracadie, au Nouveau-Brunswick. Image reproduite à partir de la référence 13

docteur John J. Prendergast, léprologue au National Leprosarium de Carville (Louisiane), rapporta en 1940 que $29 \%$ des patients étaient aveugles, ou presque, d'un œil ou des deux yeux (2).

Le traitement de la lèpre généralement recommandé pendant plusieurs années se limitait à l'huile de chaulmoogra (3), un arbre du Sud asiatique. Ce traitement avait été introduit dans la colonie de Tracadie vers 1901 par le docteur Alfred Corbett Smith (1841-1909). Selon ce dernier, il apportait des résultats encourageants (4). Après le départ du docteur Smith pour le lazaret de l'île D'Arcy, en Colombie-Britannique, le traitement demeura recommandé par son remplaçant, le docteur Joseph Damase Pagé (1861-1938), alors délégué du Service de quarantaine de l'Agence de santé publique du Canada. Administrée par voie interne à doses progressives ou par voie externe sous forme de pansements, l'huile de chaulmoogra resta le mode de thérapie de choix des léprologues. Jusqu'en 1924, on proclama les résultats merveilleux obtenus des injections hypodermiques ou intraveineuses d'huile d'éthylester du chaulmoogra (5). La découverte la plus utile et la plus spectaculaire survint en 1963, avec l'introduction des sulfamides et de leurs dérivés comme traitement révolutionnaire de la lèpre. Ce traitement fut suivi par une polychimiothérapie avec l'utilisation, le plus souvent, de la rifampicine, de la Disulone, du Lamprène ou de la clofazimine, de l'éthionamide et du prothionamide. Un fait fort intéressant a marqué le traitement de la lèpre au tournant du présent millénaire. La thalidomide serait alors devenue le médicament de choix, bien qu'elle demeure formellement contre-indiquée aux femmes enceintes à cause de sa grande tératogénie (6).

Les publications médicales soulignent à plusieurs reprises la présence de lazarets au Canada. Nous en retenons quatre qui semblent bien illustrer la problématique de ces établissements d'isolement des victimes de la lèpre, aux extrémités est et ouest du pays. Parmi les premières publications canadiennes sur le sujet, le Canada Lancet fait remarquer à deux reprises, au cours de l'année 1883, l'existence d'une léproserie à Tracadie, dans les comtés de Gloucester et de Northumberland du Nouveau-Brunswick $(7,8)$. La publication canadienne la plus documentée sur les lazarets à la fin du XIX ${ }^{\mathrm{e}}$ siècle est le Dominion Medical Monthly, où l'on trouve aussi une description détaillée de la colonie de l'île D'Arcy (9), en Colombie-Britannique

\section{LES LÉPROSERIES DE L'ÎLE DE SHELDRAKE (1844-1848) ET DE TRACADIE (1848-1965), AU NOUVEAU-BRUNSWICK}

Retour sur le passé. Tout a commencé par la venue de deux marins norvégiens débarqués d'un vaisseau, appelé La Florida, lors d'une escale à l'automne 1815 à Caraquet (NouveauBrunswick), sur les rives de la Baie des Chaleurs (10). Les deux hommes y passèrent l'hiver après avoir été accueillis charitablement dans une famille acadienne nommée Benoît, en dépit de la répugnance de leur corps couvert d'ulcères. Quelques années plus tard, certains membres de la famille, notamment Ursule Benoît (née Landry) qui avait pris soin de laver leur linge, développèrent la maladie. Elle en mourut en 1828 et son mari, Joseph Benoît, succomba à son tour en 1834. La maladie emporta aussi deux soeurs d'Ursule, Isabelle et Françoise, et plusieurs de leurs enfants.

Le docteur Joseph-Charles Taché (1820-1894), de l'Université Laval de Québec, et le docteur Alfred Corbett Smith (1841-1909), membre du Conseil médical du NouveauBrunswick et chargé de l'institution de Tracadie, confirmèrent tout simplement la contagion de la maladie en signalant qu'un jeune homme du nom de Francis Soney, alors en bonne santé, aurait contracté la lèpre cutanée après avoir porté sur une épaule éraflée le cercueil d'Ursule Benoît d'où suintait un liquide visqueux (11). Il mourut onze ans plus tard. Trente cas furent signalés dans les deux décennies qui suivirent, et l'horrible maladie devint endémique pendant deux générations au sein de la colonie canadienne-française. La plupart des lépreux étaient d'ascendance française, mais on compta aussi quelques victimes d'ascendance anglaise, irlandaise ou écossaise.

En 1844, inquiète de la tournure des événements, la population de la région demanda au lieutenant-gouverneur la construction d'un hôpital. On acquiesça à cette requête, d'ailleurs bien justifiée, le 31 mai 1844, en aménageant un lazaret sur l'île de Sheldrake, à l'embouchure de la rivière Miramichi, où 12 malades furent isolés. Tout alla bien un certain temps, mais les choses se gâtèrent le 16 juillet de l'année suivante, lorsque le Sheldrake Island Hospital fut brûlé par les lépreux eux-mêmes, impatients de recouvrer leur liberté. Sur 18, quatre s'évadèrent et les 14 autres, pris au piège, durent s'accommoder de l'espace évidemment réduit de la bâtisse adjacente au lazaret, qui servait de salle de lavage et avait été épargnée par le feu.

L'affaire remonta jusqu'aux instances gouvernementales et, en 1849, les intervenants acceptèrent de construire un nouvel hôpital à Tracadie même (voir figure 1), dans l'intention d'assurer à la fois la sécurité du public et le confort des malades, tout en veillant à ce que ceux-ci puissent communiquer facilement avec leurs proches. Hélas, de 1849 à 1861, l'enfer déjà vécu par les malades sembla se raviver. L'hôpital devint un véritable centre de détention, dirigé d'une main de fer. Le docteur Frederick Lawson Whitehead (1909-1994), d'East Riverside (NouveauBrunswick), révéla qu'en 1861, après avoir pris la relève à la direction de l'institution, le docteur James Nicholson (18341865) mit un frein au pouvoir despotique de ses prédécesseurs, dont il décrit ainsi la dureté : « les lépreux étaient chassés comme des bêtes sauvages, attachés à des poteaux avec des crochets de fer, menottés et entourés d'un mur de 12 pieds de hauteur » (12).

Avec la venue du docteur Nicholson, la terreur de l'isolement disparut. On s'étonnera cependant dans une certaine mesure d'apprendre que, par la suite, les patients se présentaient d'eux-mêmes pour être examinés et hospitalisés (13). Néanmoins, on constata plus tard que peu de personnes 


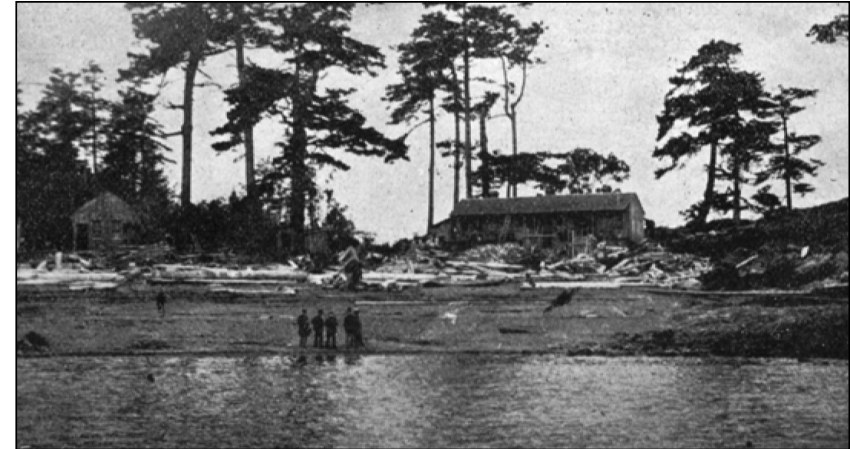

Figure 2) Lazaret à l'île D'Arcy, vu du bateau, Vancouver, en Colombie-Britannique. Image reproduite à partir de la référence 9

visitaient les malades ou s'enquéraient de leur état. D'une part, la lèpre était souvent considérée comme une honte dans la famille ou une malédiction de Dieu, et l'on s'efforçait d'oublier les lépreux. D'autre part, les malades eux-mêmes perdaient intérêt au monde extérieur et devenaient indifférents aux affaires familiales (14). Il faut donc comprendre que les malades affligés, souvent originaires des milieux les plus pauvres parmi les fermiers, les pêcheurs et les bûcherons, s'y trouvaient logés plus confortablement qu'à la maison. Souvent défigurés et infirmes à cause des funestes manifestations de la maladie, ils en venaient à ne plus souhaiter quitter l'institution. Il va sans dire que l'endroit revêtait l'allure d'une véritable antichambre de la mort.

Une anecdote pathétique évoque une image inoubliable. Un beau matin, trois jeunes garçons de 11 à 15 ans, de bonne allure et au regard intelligent, se présentèrent au lazaret. On les confina aussitôt dans l'unique chambre réservée aux hommes; ils furent séquestrés, coupés du monde extérieur à tout jamais condamnés. Ils vécurent leur jeunesse, leur vie d'adulte et leur vieillesse dans une société isolée, formée uniquement d'autres victimes de la lèpre. Rien à faire, aucune tâche, aucune occupation, aucun amusement, aucune ressource. Rien pour marquer le temps, si ce n'est l'arrivée d'autres lépreux comme eux et regarder mourir leurs compagnons (15).

Le 18 septembre 1868 demeure une date importante. Seize religieuses hospitalières de Saint-Joseph, venues de Montréal et ignorant la peur de la contagion, répondirent avec un empressement héroïque à l'appel suppliant d'hommes et de femmes frappés du mal terrifiant de la lèpre et mis au ban de la société. Elles acceptèrent la mission et offrirent généreusement leurs services, prenant en charge l'administration de l'hôpital, veillant à la distribution adéquate des médicaments aux malades, à la visite des salles pour s'assurer du bon traitement des malades et s'assurant du bon entretien des lieux, quitte à embaucher deux femmes de ménage. Elles y restèrent jusqu'en 1965, au départ du dernier patient (16).

\section{LES COLONIES DE LÉPREUX DE L'ÎLE D'ARCY (1891-1924) ET DE L'ÎLE DE BENTINCK (1924-1957) EN COLOMBIE-BRITANNIQUE}

Tout a commencé en 1891. Cinq lépreux, qui avaient esquivé la vigilance des agents d'immigration à William Head, furent découverts dans le Chinatown par la police de Victoria au cours d'une ronde de routine. Ils étaient cachés dans un magasin de la rue Fisgard. Puisque la lèpre était, bien sûr, considérée comme une maladie transmissible, il fallait prendre des

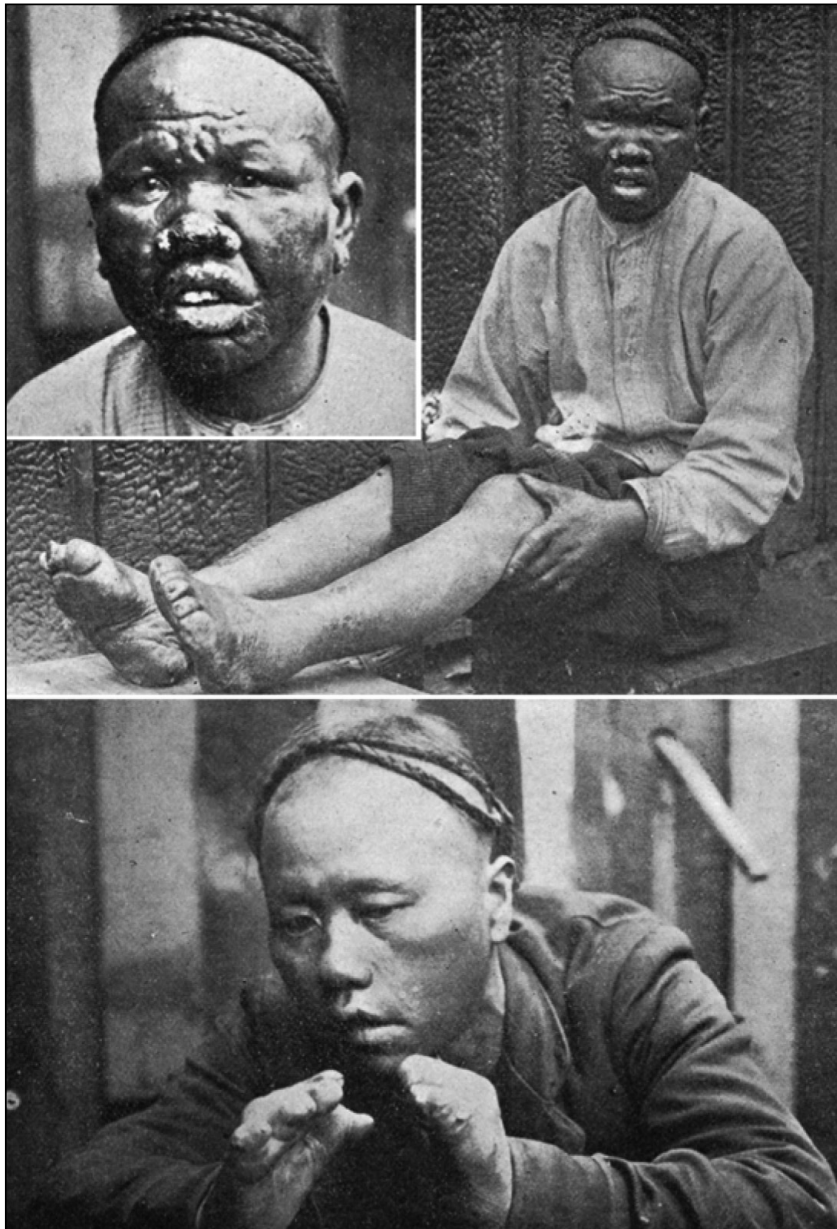

Figure 3) Lazaret à l'île D'Arcy de Vancouver, en ColombieBritannique. Image reproduite à partir de la référence 9

mesures rigoureuses pour isoler aussi vite que possible les lépreux de la population saine. Afin de prévenir une panique générale, les autorités municipales de Victoria exproprièrent aussitôt l'île D'Arcy pour y établir une léproserie en construisant en toute hâte une cabane, (voir figure 2) en construisant comprenant six chambres distinctes pour loger les lépreux. Mais où se trouvait cette île isolée ? L'île D'Arcy, couverte de pins, de cèdres et d'épinettes, se situe dans l'océan Pacifique à environ 30 kilomètres au large de l'île de Vancouver. Et qui étaient ces malheureux pour être isolés de la sorte ? Le commerce du Royaume-Uni avec les pays de l'Orient avait entrainé un énorme influx d'immigrants asiatiques, particulièrement des Chinois venus travailler à la cons-truction du chemin de fer canadien. Ainsi, l'importation de la lèpre sur le littoral du Pacifique en Colombie-Britannique était définitivement attribuable à l'immigration des Orientaux.

La monotonie et la désolation de la vie sur cette île sont difficilement descriptibles. Rien ne changeait dans le petit monde de ces misérables créatures, si ce n'est le sempiternel retour des saisons, la détérioration constante de leur état de santé, (voir figure 3) et le poids alourdissant de la lassitude et de la dépression. Une longue vie, mais sans espoir ! Ces détenus avaient beau entretenir eux-mêmes un jardin verdoyant où poussaient de splendides légumes : pommes de terre, laitue, oignons et choux, sans parler du verger prometteur de pommes, de pêches et d'abricots ni de la clameur attrayante des 
mouettes, il n'en demeurait pas moins que l'île était une véritable prison sans barreaux. Victimes de leur maladie, ces prisonniers en étaient venus à se considérer eux-mêmes comme des criminels (13). La seule animation, en contraste avec le reste de l'île, venait de la basse-cour où s'activait un troupeau d'environ 150 poulets et d'une trentaine de canards.

Tous les trois mois dès la fondation, puis toutes les semaines à partir de 1905, les agents de la santé laissaient sur la plage de la nourriture et des vêtements pour répondre à leurs besoins, sans parler des cercueils pour que les vivants puissent enterrer les morts. Incidemment, un seul homme «blanc » fut isolé dans cet établissement. Il fut vite rejeté par les autres malades, tous Asiatiques. Une communauté aussi homogène exigeait évidemment de chaque membre une similitude fondamentale et une dépendance mutuelle pour survivre. À la mort de l'homme «blanc », inévitable par suite de son isolement et de la négligence des autres lépreux, ces derniers refusèrent avec obstination de l'enterrer jusqu'à ce que les autorités menacent sévèrement de leur restreindre la nourriture (9).

\section{BIBLIOGRAPHIE}

1. Anonymous. Leprosy in Canada. CMAJ 1924;14:879.

2. Prendergast JJ. Ocular leprosy in the United States.

Arch Ophthalmol 1940;23:112-37.

3. Editorial. The Lepers at Tracadie. Montreal Med J 1905;34:207.

4. News. Four Deaths at Lazaretto, Tracadie, N.B. Dom Med Month 1901;16:95.

5. Pagé JD. Leprosy in Canada. Can Pract 1924;49:363-8.

6. Boggild AK, Keystone JS, Kain KC. Leprosy: A primer for Canadian physicians. CMAJ 2004;170:71-8.

7. Reports of Societies. Leprosy at Tracadie. Can Lancet 1883;16:30.

8. Reports of Societies, Canadian Medical Association. Leprosy in New Brunswick. Can Lancet 1883;16:48-50.

9. Hall E, Nelson J. The lepers of D'Arcy Island. Dom Med Month 1898;11:233-9.

10. Meiklejohn ML. The early hospital history of Canada, 1535-1875. I. French Régime. Montreal Med J 1910;39:297-320.
Aussi étrange que cela puisse paraître, ces Asiatiques méprisaient le riz et la farine et, encore plus incroyable, ils rejetaient le thé chinois avec dédain, mais ils préféraient le gin et le whisky ! Whisky, opium ou pas, l'endroit demeurait intenable. Illustration éclatante : un jour un agent de la Santé, dans sa tournée du Chinatown de Vancouver, à la recherche cette fois de "varioleux », découvrit par hasard un lépreux. Un interprète fit comprendre au malade qu'il devait se tenir prêt à être déporté le lendemain sur l'île D'Arcy. L'homme se suicida la nuit suivante avec une dose massive d'opium (9).

En 1905, on apprend que les malades de la colonie auraient tous été déportés vers un hôpital presbytérien de Canton, en Chine (17). Il n'en demeure pas moins qu'en 1924, neuf lépreux furent transférés du lazaret de l'île D'Arcy à celui de l'île Bentinck pour les rapprocher de la station médicale de quarantaine de William Head (18). Le lazaret de l'île Bentinck fut finalement fermé à la mort du dernier lépreux en 1957.

Fin d'une époque tragique des grands moments de notre histoire médicale canadienne, à conserver dans notre mémoire du passé !

11. Taché JC, Smith AC. Leprosy in New Brunswick. Can Lancet 1886;18:152-3.

12. Whitehead FL. Leprosy in New Brunswick: The end of an era. Can Med Assoc J 1967;97:1299-300.

13. Kelly CM. An account of Canadian leprosy. Montreal Med J 1909;38:387-92.

14. Miscellaneous. Leper institute in New Brunswick. Can Lancet 1910;63:792-3

15. Heagerty JJ. Four centuries of medical history in Canada. Leprosy. Toronto: The Macmillan Company of Canada Limited, 1928:161-78.

16. Religieuses hospitalières de Saint-Joseph. $<$ http://personal.nbnet.nb.ca/rhsjnda/Page3.html> (version à jour le 22 février 2008).

17. Johnton P. BC's "island of death" marked a sad chapter in Canada's medical history. CMAJ 1995;15:951-2.

18. Pagé JD. Leprosy in Canada. CMAJ 1924;14:824-5. 


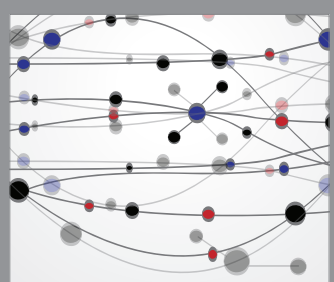

The Scientific World Journal
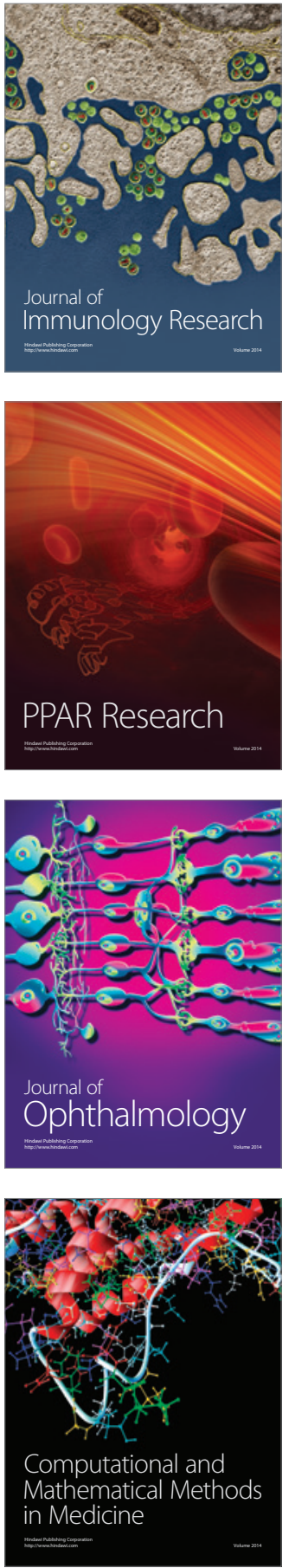

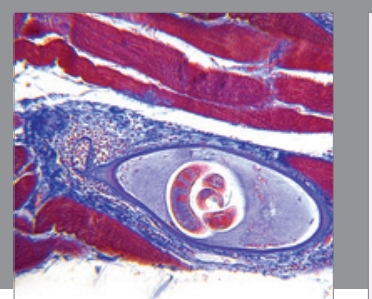

Gastroenterology Research and Practice

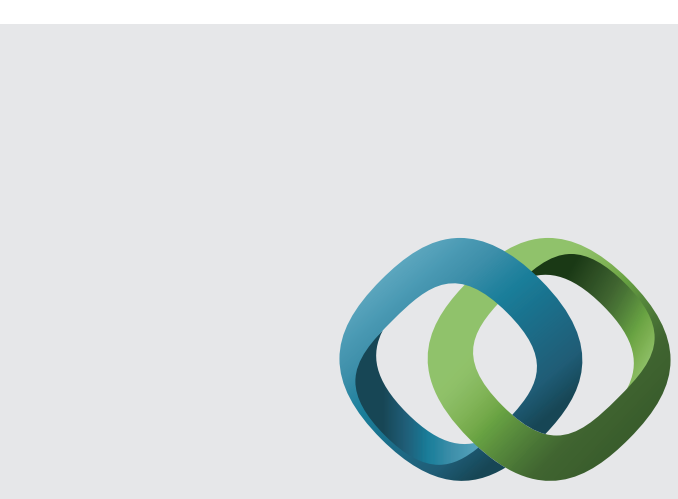

\section{Hindawi}

Submit your manuscripts at

http://www.hindawi.com
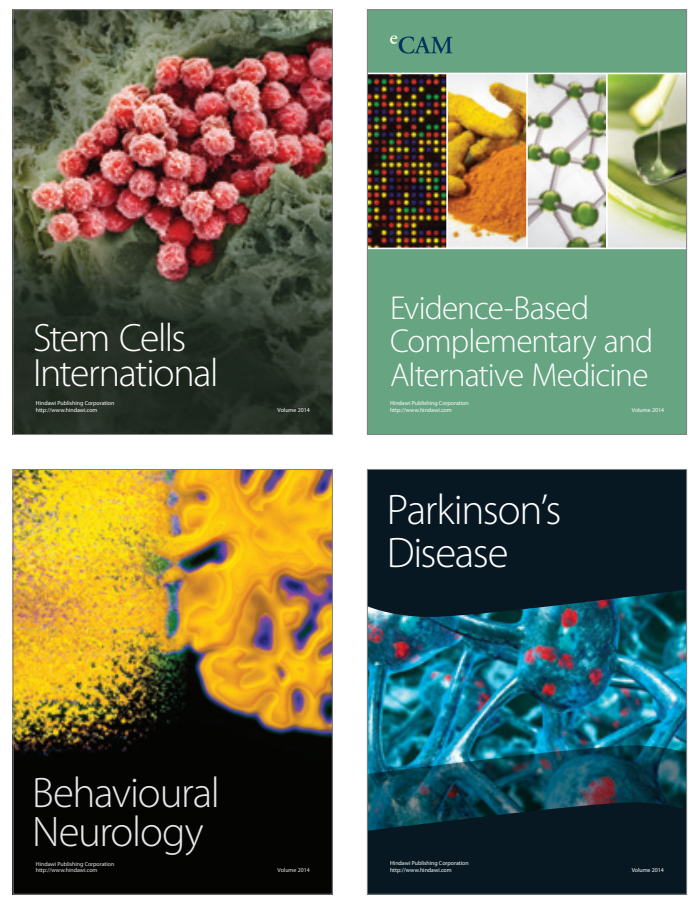
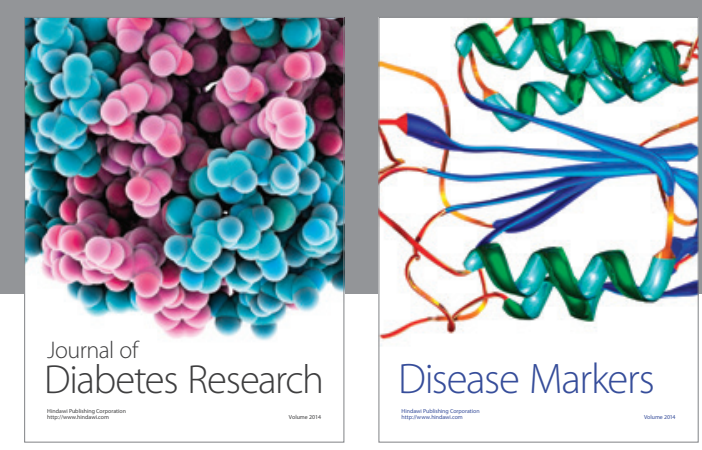

Disease Markers
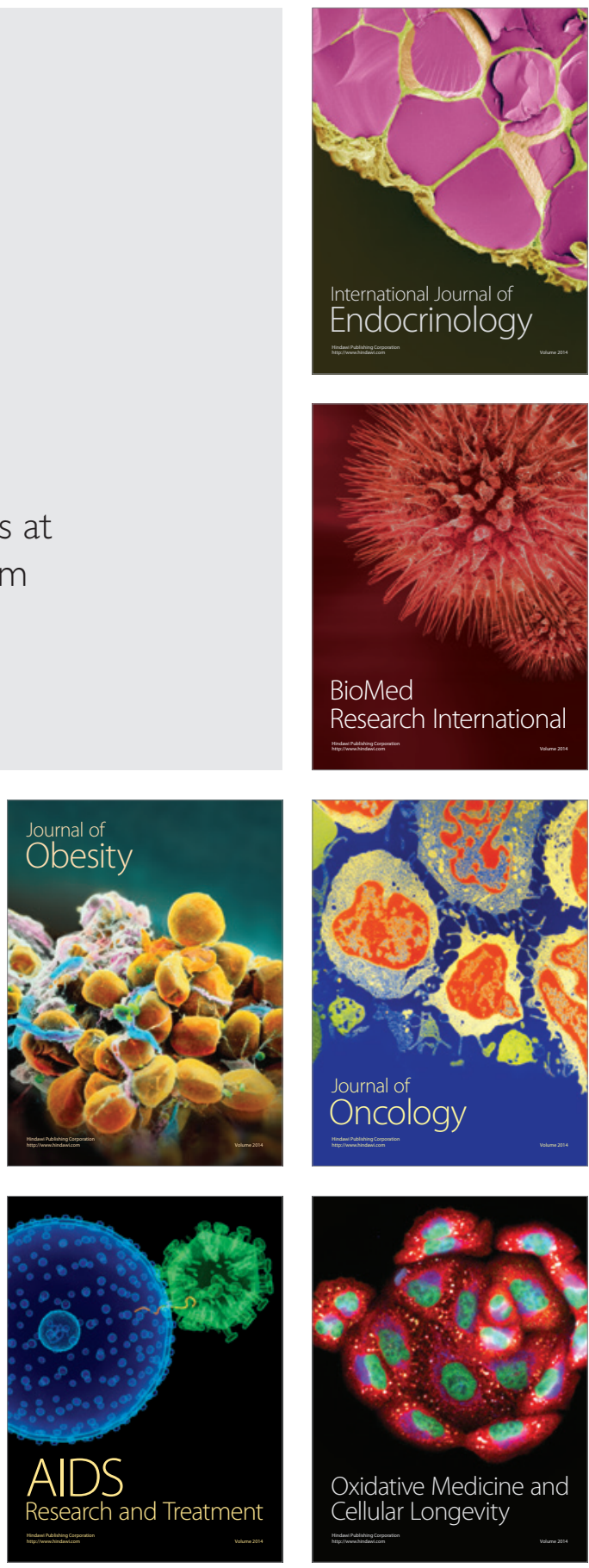